\title{
Metafunción TEXTUAL: BASE PARA LA ALFABETIZACIÓN ACADÉMICA EN DERECHO CONSTITUCIONAL
}

\author{
Textual Metafunction: A Basis for Academic Literacy in Constitutional Law
}
MÉTAFONCTION TEXTUELLE : UN FONDEMENT POUR LA LITTÉRACITÉ ACADÉMIQUE AU DROIT CONSTITUTIONNEL

\author{
llene Rojas García \\ Ed. D., Fundación Universidad del \\ Norte, Barranquilla, Colombia. \\ Profesora transitoria, Facultad de \\ Educación, Universidad Tecnológica \\ de Pereira, Colombia. \\ dirojas@uninorte.edu.co \\ https://orcid. \\ org/0000-0001-5203-3988
}

Este artículo sintetiza los resultados de la investigación "Metafunción textual y lectura para el aprendizaje de contenidos en la asignatura de derecho constitucional", realizada cuando la autora era becaria de doctorado en Educación de la Universidad del Norte (Barranquilla, Colombia), 2018.

\section{RESUMEN}

El artículo atiende al imperativo de la formación para la lectura en las disciplinas en el ámbito de la educación superior. Se seleccionaron cuatro textos, usados como material de estudio en el aula de Derecho Constitucional de la Universidad del Norte, en Colombia. En ellos se describen los patrones de Tema-Rema, así como el método de desarrollo y el sistema de PERIODICIDAD. Desde allí se configuró la descripción del género explicativo de la doctrina del derecho. Se constata que la textura discursiva puede ocasionar dificultades en el aprendizaje de los estudiantes cuando se presentan patrones no congruentes o construcciones complejas. Por ejemplo, en el nivel semántico-discursivo, la información progresa mediante cláusulas subordinadas y, en ocasiones, el Tema no corresponde con la información conocida. Con respecto al nivel léxico-gramatical, la presencia de términos referenciales restringe la lectura lineal y las nominalizaciones aumentan la densidad conceptual. Además, el Tema marcado interrumpe el flujo del discurso, mientras el uso del Tema interpersonal exige un lector experto. Estos resultados constituyen la base para una propuesta didáctica de lectura en un curso de competencias comunicativas con énfasis en derecho constitucional; a través de la pedagogía de género, se evidencian los patrones discursivos que intervienen en el aprendizaje.

Palabras clave: alfabetización académica; derecho constitucional; lingüística sistémico-funcional; metafunción textual; pedagogía de género.

\section{Abstract}

This article addresses the imperative of instruction on reading in the disciplines in higher education. Four texts used as study material in a Constitutional Law course at Universidad del Norte, Colombia, were analyzed. The study describes the Theme-Rheme patterns, as well as the method of development and PERIODICITY. From there, a description of the explanatory genre of the doctrine of Law was made up. The analysis showed that discursive texture can cause trouble to students when there are non-congruent patterns or complex constructions. For instance,

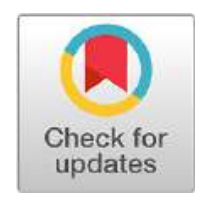

Recibido: 2020-06-08 / Aceptado: 2020-10-02 / Publicado: 2021-01-30

https://doi.org/10.17533/udea.ikala.v26n01a01 
at the discourse-semantic level, the information progresses through subordinate clauses and sometimes the topic does not correspond with the known information. In regard to the lexical-grammatical level, the presence of referential terms restricts linear reading and nominalizations increase conceptual density. Besides, the marked Theme interrupts the flow of speech, while the use of the interpersonal Theme requires an expert reader. These results constitute the basis for a didactic reading proposal in a communicative competences course with an emphasis on constitutional law; through genre-based pedagogy, the discursive patterns that intervene in learning are evidenced.

Keywords: academic literacy; systemic-functional linguistics; textual metafunction; constitutional law; genre-based pedagogy.

\section{RÉSUMÉ}

L'article aborde la nécessité d'instruction dans la lecture dans les disciplines dans un contexte universitaire. Quatre textes utilisés comme matériel d'étude dans la classe de Droit Constitutionnel de l'Universidad del Norte, en Colombie, ont été sélectionnés. Ils décrivent les modèles Thème-Rhème, ainsi que la méthode de développement et le système de PÉRIODICITÉ. À partir de là, la description du genre explicatif de la doctrine du droit a été configurée. On constate que la texture discursive peut causer des difficultés dans l'apprentissage des élèves lorsqu' il y a des motifs non congruents ou des constructions complexes : au niveau sémantique-discursif, l'information progresse à travers des clauses subordonnées et parfois le sujet ne correspond pas à l'information connu. Au niveau lexico-grammatical, la présence de termes référentiels restreint la lecture linéaire et les nominalisations augmentent la densité conceptuelle; le Thème marqué interrompt le flux de la parole, tandis que l'utilisation du Thème interpersonnel nécessite un lecteur expert. Ces résultats constituent la base d'une proposition de lecture didactique dans une classe de compétences communicatives avec un accent sur le droit constitutionnel; grâce à la pédagogie du genre, les schémas discursifs qui interviennent dans l'apprentissage sont mis en évidence.

Mots clés : alphabétisation académique; linguistique systémique-fonctionnelle; métafonction textuelle; droit constitutionnel; pédagogie du genre. 


\section{Introducción}

Halliday (1993) describe el aprendizaje como un proceso de construcción de significados por medio del lenguaje o, lo que es lo mismo, interpreta que la capacidad de reconstrucción simbólica de la realidad determina las posibilidades de acceso al saber. En la educación superior, esta premisa adquiere especial relevancia, puesto que se espera del estudiante mayor autonomía para aprender leyendo; sin embargo, es evidente que esta habilidad requiere un proceso de alfabetización académica, es decir, aprender a usar el discurso propio de la disciplina para acceder a sus significados (Carlino, 2013; García-Parejo et al., 2017; Ignatieva y Rodríguez, 2016; Moyano, 2007, 2010; Narvaja et al., 2004;). La alfabetización académica, liderada por los docentes de lengua, requiere, en un primer momento, que estos amplíen el conocimiento de los géneros discursivos usados en las diferentes áreas de formación profesional, para, luego, implementar estrategias didácticas orientadas a que el estudiante adquiera el dominio del texto que le facilite el acceso al saber (Álvarez, et al., 2018; Dreyfus et al., 2016).

El conocimiento de los géneros disciplinares y su enseñabilidad en el aula universitaria son los objetivos de la investigación "Metafunción textual y lectura para el aprendizaje de contenidos en la asignatura de derecho constitucional"; en ella se abordaron cuatro capítulos, tomados de dos libros que se proponen como material de apoyo por la docente de la asignatura Derecho Constitucional. El desarrollo de este trabajo tuvo como base la lingüística sistémicofuncional (LSF) (Halliday, 1994; Martin y Rose, 2007, 2008; Rose y Martin, 2012), desde donde se retoma el concepto de género como piedra angular para los estudios del discurso, además de los conceptos de PERIODICIDAD, método de desarrollo y Tema como elementos de la metafunción textual.

Los datos son analizados según sus niveles de congruencia, para determinar aspectos que inciden en el aprendizaje del contenido curricular en el ámbito universitario de la formación de abogados. Los resultados se comentan en función de una propuesta para la didáctica de la lectura, que tiene como base la pedagogía de género, formulada por la Escuela de Sidney (Martin y Rose, 2008; Rose y Martin, 2012).

\section{Marco teórico}

La LSF permite relacionar directamente la materialidad del texto con sus significados, en el marco de un contexto cultural; en esta medida, entiende la lectura como un proceso de participación activa y consciente en las diferentes esferas de la realidad social. Siguiendo a Martin (1992, p. 25), el género se define como una actividad a partir de la cual los hablantes se relacionan como miembros de una cultura; en este sentido, al interior del ámbito universitario, el dominio de los géneros especializados hace posible acceder a las dinámicas propias del actuar académico, base de la formación profesional.

Martin (2016) posteriormente define el género como una configuración recurrente de significados, que orienta las elecciones de campo, tenor y modo; el conjunto de estos significados es denominado "registro". El campo se refiere a aquello que está sucediendo en la situación comunicativa; el tenor corresponde al rol de los sujetos que toman parte en la actividad sociosemiótica, y el modo permite realizar y concretar, en el texto, las dos variables anteriores (Halliday, 1994).

En correspondencia con las opciones del registro, Halliday (1994) distingue tres metafunciones del lenguaje, que transversalizan todo el modelo del lenguaje: se trata de las metafunciones ideacional, interpersonal y textual. La primera organiza la experiencia y ayuda a construir el conocimiento sobre el mundo; la segunda contribuye con el mantenimiento de las relaciones interpersonales y define los roles en la interacción, mientras que la tercera permite traducir la experiencia y las condiciones de interacción en un discurso coherente.

Estos significados, de carácter sociosemiótico, se realizan en el lenguaje en forma de cascada, desde el estrato semántico-discursivo, pasan por 
Tabla 1 Estratos del contexto y el lenguaje

\begin{tabular}{|c|c|c|c|c|}
\hline \multicolumn{2}{|c|}{ Estrato sociosemiótico } & \multirow[t]{2}{*}{ Metafunciones } & \multicolumn{2}{|c|}{ Sistemas de representación } \\
\hline Género & Registro & & $\begin{array}{c}\text { Estrato } \\
\text { semántico-discursivo }\end{array}$ & Estrato léxico-gramátical \\
\hline \multirow{6}{*}{$\begin{array}{l}\text { Patrones recurrentes } \\
\text { de significado }\end{array}$} & Campo & Experiencial & IDEACIÓN & Transitividad \\
\hline & & Ideacional & Conjunción & Taxis \\
\hline & Tenor & Interpersonal & Valoración & MODALIDAD \\
\hline & & & Negociación & Polaridad \\
\hline & Modo & Textual & Periodicidad & TEMA \\
\hline & & & IDENTIFICACIÓN & INFORMACIÓN \\
\hline
\end{tabular}

Fuente: Adaptado de Eggins y Martin (2003)

el estrato léxico-gramatical y finalizan en el estrato grafo-fonológico.

En cada estrato del lenguaje, se configuran sistemas de realización del significado, que corresponden con cada metafunción (Martin y Rose, 2007). En este sentido, en el estrato semántico-discursivo, la metafunción ideacional configura el sistema de IDEACIón que se enfoca en el contenido del discurso, y determina las relaciones lógicas entre estos contenidos que se realizan mediante el sistema de CONJUNCIÓN. La metafunción interpersonal se codifica por medio del sistema de VALORACIÓN, donde se negocian las actitudes y la fuerza de los sentimientos involucrados; se incluye, además, el sistema de NEgociación, que resalta la interacción donde los hablantes adoptan y asignan roles. Con respecto a la metafunción textual, el sistema de PERIODICIDAD organiza el discurso como pulsos de información que anuncian el contenido y luego consolidan los datos; finalmente, el sistema de IDENTIFICACIÓN, se refiere al seguimiento de los participantes a lo largo del texto.

En la Tabla 1 se sintetizan los estratos del contexto (género y registro) y del lenguaje (semántico-discursivo y léxico-gramatical).

La investigación que se reporta en este artículo se enfocó específicamente en el análisis desde la metafunción textual, específicamente desde el sistema de PERIODICIDAD, el método de desarrollo y los patrones de Tema. Se trata del aspecto de mayor factibilidad en la comprensión de la cláusula, ${ }^{1}$ puesto que abre una ventana a las otras metafunciones (Halliday, 1994).

Martin y Rose (2007) explican el sistema de PERIODICIDAD como picos de información a lo largo del texto, que organiza los significados ideacionales e interpersonales, es decir, el párrafo puede iniciar con una cláusula que anticipa su contenido, conocida como "hiperTema", y finaliza con otra cláusula que sintetiza el texto desde otra perspectiva, es decir, el "hiperNuevo". En síntesis, "el hiperTema indica dónde vamos a estar en una fase, mientras que el hiperNuevo señala dónde estuvimos" (Martin y Rose, 2007, p. 195).

Asimismo, es posible determinar el campo de la actividad a la que alude el texto a partir de la observación de la sucesión de elementos léxicos seleccionados como Tema de la cláusula, lo que en inglés ocurre en posición inicial, mientras que en español se realiza por concordancia (Moyano, 2016); se trata de lo que Fries (1983) denomina el "método de desarrollo” (MoD). Martin (1992) señala que el MoD proporciona a los interlocutores datos en los cuales pueden apoyarse, una orientación, algo a lo que volver; por lo general, corresponde con el asunto

1 La cláusula es entendida como la unidad básica del estrato léxico-gramatical, que construye los significados de las tres metafunciones en sistemas relativamente independientes: ideacionales, interpersonales y textuales (Halliday, 1994). 
del discurso, de manera que ayuda a mantener la unidad textual.

Eggins (2002) señala que la reiteración del Tema a través de cadenas de identidad y semejanza tiene el efecto de mantener un fuerte enfoque tópico, al tiempo que evita la redundancia; pero un texto en el cual el Tema nunca varía, sería molesto y no tendría una dirección clara. Esta variación puede generarse a partir de un cambio en el elemento temático, estableciendo una conexión con el discurso, no desde la reiteración del Tema anterior, sino por conexión con el Rema de la cláusula precedente, de manera que se posibilite el progreso de la información. En este sentido, mantener la secuencia temática genera la continuidad del flujo del discurso, mientras que los cambios en el MoD configuran la progresión. Cabe resaltar que el $\mathrm{MoD}$ es susceptible al género, es decir, cuando se cambia la identidad del Tema, aparece una nueva fase en el texto; por tanto, se hace posible usar únicamente el Tema de las cláusulas para reconocer las etapas de la estructura genérica.

En el nivel léxico-gramatical, Halliday (1994) asume la cláusula con el carácter de mensaje, como la información que organiza y contribuye al flujo del discurso. En este sentido, define la función gramatical Tema en tanto "elemento que sirve como punto de partida del mensaje; es el que localiza y orienta la cláusula en su contexto" (p. 37). Para el caso del español, Moyano (2016) señala que el Tema se realiza mediante concordancia, de manera que el Tema se manifiesta mediante el participante concordante, aunque se encuentre elidido, pues es posible inferirlo no solo de las cláusulas precedentes sino también de la flexión verbal. Con respecto a los casos de inversión en el orden participante concordante - Proceso, es lógico aplicar el mismo criterio de recuperación léxica y trabajar el Tema desplazado.

Para estudiar el sistema de TEMA de la metafunción textual, es necesario retomar algunos aspectos de la metafunción ideacional, también del nivel de la cláusula, esto es, el sistema de TRANSITIVIDAD. El punto de convergencia entre ambas metafunciones permite delimitar el Tema no marcado como un participante (grupo nominal o pronombre explícito o elidido), el Tema marcado como una circunstancia (de modo, tiempo, lugar o de ángulo) y cláusulas sin Tema como aquellas que incluyen verbos existenciales o impersonales.

Para determinar las posibilidades de aprendizaje que pueden resultar de las elecciones semántico-discursivas y léxico-gramaticales, en esta investigación se adopta el concepto de congruencia. Halliday (1993) y Halliday y Martin (1993) señalan que la congruencia es propia del discurso cotidiano, y se complejiza en el discurso científico, ya que requiere formas de expresión más abstractas, metafóricas y empaquetadas, capaces de convertir la reflexión de la ciencia en significado lingüístico. Por tanto, mientras más se aleje el discurso de las formas congruentes del lenguaje cotidiano, mayores dificultades puede tener el lector para asimilar el contenido e identificar la función de las elecciones discursivas.

A nivel de la metafunción textual, la congruencia está determinada por tres factores: la existencia de una cláusula hipertemática, que anticipe el contenido del párrafo; el conjunto de Temas, desde los cuales se construya el MoD, y la convergencia entre el Tema y el asunto de la cláusula. Cuando estos patrones se alteran, será necesario crear estrategias pedagógicas tendientes a lograr, en el estudiante, la capacidad para establecer las relaciones lógicas que existen entre los distintos segmentos textuales y las pistas que ofrece el texto para seguir el hilo del discurso.

De los postulados de la LSF, Rose y Martin (2012) derivan un ciclo de enseñanza del lenguaje que trabaja sobre los textos del currículo. A partir de allí, los autores presentan la enseñanza de la lectura como una serie de capas que corresponden con los estratos del lenguaje. Sugieren iniciar con el estudio del género, para preparar a los estudiantes en los conocimientos contextuales que requieren antes de la lectura. En el siguiente nivel se revisan los patrones discursivos, con el objeto 
de comprender el texto como unidad estructural. La etapa final se enfoca en el nivel lexico-gramatical, prestando especial atención a los patrones oracionales, para construir el significado. A través de estas fases, el docente actúa como mediador entre las estructuras textuales y las necesidades de aprendizaje de los estudiantes.

\section{Método}

La unidad de análisis para la descripción del género de los textos en Derecho Constitucional está conformada por cuatro capítulos usados para aprender en el curso de derecho constitucional: "Noción de Estado”(NE) (Naranjo, 2012, pp. 81-97), "Elementos del Estado” (EE) (Naranjo, 2012, pp. 97-149), "Constitución de los antiguos" (CA) (Fioravanti, 2011, pp. 15-32) y "Constitución medieval" (CM) (Fioravanti, 2011, pp. 33-70).

La tarea de lectura planteada por la docente disciplinar se enfocó en que los estudiantes lograran determinar, en los dos primeros textos, el concepto de Estado y sus elementos constitutivos, solicitándoles que elaboraran esquemas (lo que puede constituir otro género objeto de investigación en la alfabetización académica). Para los dos últimos textos, el trabajo se enfocó en revisar la forma como evolucionó el concepto de lo que puede ser entendido como Constitución en la Antigüedad y en la Edad Media, con la ayuda de un cuestionario.

Durante la realización de las tareas de lectura propuestas por la docente titular, se evidenció que los estudiantes tenían mayor dificultad en el desarrollo de las actividades de los textos de Fioravanti, mientras que las actividades sobre los textos de Naranjo eran resueltas sin mayores inconvenientes.

A partir de esta observación inicial, el trabajo investigativo se dirige a describir las características de la metafunción textual de los textos en cuestión, pues se considera que el reconocimiento de la estructura textual y la forma como se desarrollan las ideas en el texto cumplen un papel primordial en la enseñanza de la lectura para el aprendizaje.
Para ello, se separaron los párrafos y las cláusulas constitutivas de cada texto según el modelo propuesto por Moyano (2016), con los promedios que se presentan en la Tabla 2, promedios que permiten establecer los patrones recurrentes de configuración textual.

Tabla 2 Total de cláusulas y párrafos en los textos analizados

\begin{tabular}{llcc}
\hline \multicolumn{1}{c}{ Autor } & \multicolumn{1}{c}{ Capítulo } & $\begin{array}{c}\text { Total de } \\
\text { cláusulas }\end{array}$ & $\begin{array}{c}\text { Total de } \\
\text { párrafos }\end{array}$ \\
\hline Vladimiro & Noción de Estado & 280 & 55 \\
Naranjo & Elementos del Estado & 997 & 174 \\
Total & & 1277 & 229 \\
Maurizio & Constitución de los & 185 & 34 \\
Fioravanti & antiguos & & \\
& Constitución medieval & 402 & 96 \\
Total & & 587 & 130 \\
\hline
\end{tabular}

Posteriormente, se revisaron los Temas de cada cláusula y sus implicaciones en el significado; se avanza, luego, en la observación de la forma como estos elementos construyen el MoD. En una lectura panorámica se observa si existen cláusulas hipertemáticas en el desarrollo del flujo del discurso. Finalmente, se relacionan los parámetros recurrentes, que dan lugar a la descripción del género explicativo de la doctrina del derecho, en cuanto a la metafunción textual se refiere.

Una tercera fase del trabajo se centra en determinar la correspondencia entre la textura discursiva y las posibilidades de acceso que presenta para obtener los conocimientos especializados. Para ello, se retoman las construcciones lingüísticas halladas en los textos estudiados y se comparan con las consideradas como congruentes. Los resultados obtenidos en la fase de descripción de los textos y de evaluación de la congruencia son utilizados para orientar estrategias didácticas que siguen los parámetros propuestos por la pedagogía de género de la Escuela de Sídney (Martin y Rose, 2008; Rose y Martin, 2012). 


\section{Resultados}

Una vez analizado el género y la metafunción textual de los textos usados para aprender en derecho constitucional, se identifican los resultados que se enuncian a continuación.

\section{Género}

Los textos objeto de estudio se inscriben en los géneros explicativos, descritos por Martin y Rose (2008). Se trata de géneros característicos del lenguaje de la ciencia, orientados a esclarecer los hechos de la realidad, por medio de reportes descriptivos, clasificatorios y composicionales, así como explicaciones de causas y efectos.

La información en cada uno de estos géneros se realiza a través de fases, que responden al propósito comunicativo. Por ejemplo, el reporte descriptivo inicia con la presentación del fenómeno, para luego señalar sus características, mientras que la explicación factorial presenta un hecho y las causas que lo generan.

Sin embargo, es necesario resaltar que los textos objeto de investigación constituyen entidades macrogenéricas, es decir, se realizan mediante fases que en sí mismas pueden constituir géneros. En este sentido, y especialmente en los textos de Naranjo (TN), se encuentran reportes descriptivos y clasificatorios, mientras que en Fioravanti (TF) es posible hallar, además, patrones argumentativos.

Con respecto al registro (como estrato de realización del género), ambos textos demuestran su inserción en el campo del derecho constitucional, en tanto desarrollan aspectos relacionados con el Estado (definición y componentes, en TN) y con la Constitución (evolución del pensamiento político constitucional, en TF).

En relación con el tenor, los TN tienen una intención didáctica, de modo que el autor se presenta como guía en el aprendizaje, segmentando su explicación en bloques semánticos y ayudando al lector con ejemplos y aclaraciones de los conceptos considerados ajenos a su realidad. Por su parte, los TF se escriben para un público de mayor experticia; su interés es presentar la existencia de un pensamiento constitucional en dos épocas, en las cuales la doctrina tradicional no suele aceptar su existencia.

En cuanto a las fases de desarrollo del contenido, Naranjo segmenta la información usando subtítulos para incluir definiciones, explicaciones ontogenéticas y clasificaciones; mientras que Fioravanti despliega el contenido por medio de un tejido de ideas, entre las cuales se establecen distintos tipos de relaciones lógicas.

\section{Periodicidad}

Desde el sistema de PERIODICIDAD, se revisaron las cláusulas que anticipan el contenido del párrafo y permiten determinar las fases en las cuales se despliega la información. En TN se encontró que un $64 \%$ de los párrafos se construyen a partir de una cláusula hipertemática, mientras que en $\mathrm{TF}$, la frecuencia se reduce a un $33 \%$.

La forma como se construyen los párrafos con cláusula hipertemática se ejemplifica en el siguiente segmento. Como se puede observar, la primera cláusula construye el contenido general (en negrilla), mientras que las siguientes lo desarrollan:

\footnotetext{
Para efectos legales, la población de un Estado la conforman todos sus habitantes, permanentes o transeúntes, nacionales o extranjeros. Casi todas las constituciones prescriben la obligación para los habitantes, nacionales o extranjeros, de vivir sometidos a la Constitución y las leyes del respectivo Estado y respetar y obedecer a las autoridades del lugar. Así lo establece el artículo $4^{\circ}$ de nuestra Carta. Ambos, pues, están sometidos por igual al orden jurídico establecido por el Estado. Existen, sin embargo, algunas diferencias, principalmente en cuanto a los derechos de que disfrutan unos y otros dentro del Estado, como se verá más adelante (Naranjo, 2012, p. 109).
}

Ahora bien, debido a que no todos los párrafos se construyen a partir de una cláusula hipertemática, se exploraron otras opciones, ajustando 
los parámetros de la definición. En un $12 \%$ de los párrafos, se encontraron cláusulas que, aunque no aparecen en posición inicial, sí permiten señalar el contenido general; estas aparecen una o dos cláusulas después de haber iniciado el segmento. En estos casos, las cláusulas iniciales actúan más bien como preámbulo, para contextualizar o crear un nexo con la información del párrafo precedente, pero no permiten predecir el contenido a desarrollar, como ocurre en el siguiente segmento:

Como sabemos, la literatura política griega utilizó frecuentemente metáforas. Pues bien, la metáfora más apropiada para comprender nuestro problema es ciertamente la del coro,_contenida en el tercer libro de la Política de Aristóteles. El coro - dice Aristóteles - puede ser cómico o trágico, aun estando compuesto por los mismos elementos que lo componen. El coro es por ello algo más que el conjunto de los elementos que en cada ocasión lo componen (Fioravanti, 2011, p. 19).

Los textos presentan un $9 \%$ de cláusulas que anticipan el contenido del párrafo de dos formas particulares: en ocasiones, resulta ser la última cláusula del párrafo anterior; otras veces, ocurre que la cláusula inicial de un párrafo alcanza para anticipar el contenido del párrafo siguiente, de modo que este último no cuenta con una cláusula hipertemática.

Con todo, continúan existiendo $15 \%$ de párrafos en TN y $45 \%$ en el texto de TF que no contienen de ninguna manera una cláusula que permita anticipar el contenido del texto. Este hecho puede ocasionar dificultades para conseguir el aprendizaje, en tanto que la cláusula hipertemática actúa como soporte para preparar al lector acerca de la información que va a ser presentada; además, suele incluir los datos clave que van a ser desarrollados posteriormente. Al no existir esta cláusula, es labor del lector encontrar una forma de sintetizar el contenido para facilitar su recuerdo y relacionarlo con el resto del texto.

\section{Método de desarrollo}

$\mathrm{Al}$ revisar el MoD en los textos objeto de la investigación, se evidencia el campo de la actividad: el Estado (TN) y la Constitución (TF). Para explicar estos conceptos, los autores recurren a distintos recursos, que también se evidencian en el MoD y permiten determinar las fases de despliegue de la información. Naranjo desarrolla el contenido de NE a partir de las siguientes fases: definiciones de Estado, evolución del concepto, diferencia con otros términos y síntesis del significado de Estado. En EE, las fases pueden ser más claras, en tanto resaltan en los subtítulos, cada uno de los cuales alude a la conceptualización de los elementos del Estado: población, territorio, poder y soberanía.

En cuanto a Fioravanti, los elementos que dan cuenta del MoD son un poco menos homogéneos y difícilmente pueden evidenciarse en los subtítulos que, además, son muy pocos. En general, las fases en CA se presentan a partir de los autores representativos del pensamiento griego y romano; al cambiar el nombre del autor, se modifica la línea temática. Para el caso de CM, Fioravanti resalta el papel del contexto social de la época como condición para comprender el pensamiento político medieval; de allí que el MoD transite entre la descripción del contexto, la explicación de conceptos y la revisión del pensamiento de autores representativos.

Si bien el MoD direcciona el asunto del texto y señala las etapas en que se presenta el contenido, un lector novel podría tener dificultades para usarlo como herramienta de lectura comprensiva, por dos razones:

1. Los términos que señalan el campo de la experiencia sobre el cual se desarrolla el texto, y que se ubican en posición inicial de la cláusula, no suelen guardar una relación de identidad (Halliday y Hasan, 1989), es decir, no se repite la misma palabra; más bien, utilizan sinónimos o merónimos, referenciales, elisiones del Tema o se amplía el grupo nominal, construyendo, incluso, cláusulas subordinadas. El lector requiere, entonces, inferir el grado de correspondencia entre los términos. 
2. En medio de las cláusulas donde se articula el $\mathrm{MoD}$, se añade información, a manera de una espiral que va tejiendo otros rasgos en el contenido del discurso, y la cual aumenta la densidad conceptual y la dificultad para establecer las relaciones lógicas entre los distintos aspectos del contenido. El lector deberá tener presente el asunto central y la forma como cada subtópico se va adhiriendo semánticamente.

A manera de ejemplo acerca de lo que ocurre comúnmente en un segmento de los textos investigados, interesa revisar el siguiente párrafo de CA de Fioravanti: ${ }^{2}$

1 Solón representa de manera emblemática al antitirano, al fundador de la constitución de los padres, que para Aristóteles es seguramente la constitución por excelencia, aquella a la que prescriptivamente se debe tender.

2 Solón fue ciertamente un gran legislador,

3 pero sobre todo [Solón] fue el árbitro del conflicto social entre pobres y ricos.

4 A favor de los primeros [Solón] promovió la liberación de las deudas más opresivas,

5 pero a favor de los segundos [Solón] negó la distribución general de las tierras como solución de la crisis. $6 \underline{\text { A través de la adopción de una serie de medidas }}$ de fuerte relevancia social e institucional,

Solón creó una constitución media en la que todos podían reconocerse con la condición de moderar sus respectivas pretensiones.

7 de esta manera [Solón] evitó convertirse en tirano, al no ponerse a la cabeza de uno de los partidos enfrentados:

8 tirano es precisamente aquel que divide la comunidad,

9 [tirano] es el rey o el legislador que traiciona a una parte de su pueblo, haciéndose cabeza de la facción contraria.

$10 \mathrm{Si}$ esto hubiera sucedido, la constitución nacida en ese contexto no poseería la virtud del punto medio.

11 Por el contrario, [la constitución nacida en ese contexto] habría sido una constitución inestable, por estar escrita según el exclusivo interés de los vencedores, y bien pronto habría degenerado en una oligarquía o en una democracia extrema.

2 La negrilla indica el Tema experiencial; la negrilla subrayada, el Tema marcado; la cursiva, el Tema textual, mientras en corchetes se presenta el Tema experiencial elidido.
El ítem léxico recurrente, que en este caso orienta el contenido del segmento (MoD) y concuerda con el proceso, se ubica en la posición inicial de las dos primeras cláusulas: va a explicarse la obra de Solón. Este ítem aparece en cinco cláusulas más, solo que, de manera elidida, en las cláusulas 3, 4, 5 y 7, y luego del Tema marcado en la cláusula 6. En esta última, el lector debe conservar, en su memoria, el complemento de modo con el que se inicia la cláusula, para relacionarlo como el mecanismo mediante el cual Solón creó la constitución media. Precisamente, este mecanismo es el que interesa resaltar en el texto, para comprender el aporte del autor en el pensamiento de los antiguos. Así, a las cláusulas que inician con el nombre de Solón, se añaden otras que dan cuenta de su obra.

El Tema marcado de las cláusulas 4 y 5 permiten construir un $\mathrm{MoD}$ subordinado que orienta la explicación sobre la acción de Solón como árbitro entre pobres (los primeros) y ricos (los segundos). Las cláusulas 8 y 9 constituyen un solo bloque de información, orientado a definir el concepto de tirano como una realidad contraria a la armonía entre los estamentos de la comunidad. Las últimas dos cláusulas forman otro bloque de información, derivado de las cláusulas anteriores que, además, establece una relación lógica de condición (cláusula 10) y oposición (cláusula 11).

Queda demostrada, entonces, las posibilidades de comprensión lectora que se pueden lograr a partir del MoD: 1) hallar el asunto central, que corresponde con el participante concordante; 2)identificar las relaciones lógicas que se establecen a lo largo del tejido discursivo, y 3) recordar el contenido que interesa abordar en el texto (¿en qué consistió la obra de Solón? - que, por demás, es objeto de evaluación en el curso de derecho constitucional-).

\section{Tema}

En este apartado, interesa revisar con mayor detalle el Tema de las cláusulas en función de sus posibilidades para el aprendizaje. 
En los textos analizados, las cláusulas que presentan Tema participante suman el $71 \%$ en TN y el $65 \%$ en TF. En los textos especializados que circulan en la universidad, los participantes suelen realizarse como metáforas gramaticales, es decir, la información de un proceso o de toda una cláusula aparece en un grupo nominal; esto permite profundizar en el contenido, pero también requiere un mayor nivel de abstracción y conocimiento del asunto.

En la cláusula que a continuación se presenta como ejemplo, tomada de CA, el autor empaqueta la información del contexto social para ubicarlo como causa de un conflicto:

La mercantilización de la polis produce también, con frecuencia de manera violenta, un creciente conflicto entre pobres y ricos, en el que los primeros reivindican formas cada vez más amplias de asistencia pública (Fioravanti, 2011, p. 15).

Obsérvese que el término "mercantilización" implica un aumento del intercambio comercial en el siglo IV a. C., por el cual se modifican las relaciones sociales, hasta ahora estables en una sociedad igualitaria. Si bien este hecho fue explicado en cláusulas anteriores, construcciones como esta pueden aumentar la dificultad del lector novel, que debe marchar al tiempo con el texto, sintetizando la información presentada para añadir nuevos datos.

Cláusulas que inician con verbos existenciales e impersonales, y cláusulas dependientes con verbos en participio ocurren en un 10\% de los casos. La dificultad que pueden presentar para un lector novel es que este tipo de procesos no permite un anclaje del contenido.

Los circunstanciales, por su parte, se realizan mediante grupos adverbiales o frases preposicionales; se encuentran antes del Tema experiencial en el $19 \%$ de las cláusulas de TN y el $24 \%$ en TF. En los TF, las Circunstancias de tiempo y de ángulo ayudan a delimitar las fases. Sin embargo, pueden interrumpir el flujo del discurso cuando se incluyen como aclaraciones previas al Tema no marcado y no para marcar discontinuidad como ocurre en el caso del inglés (Martin y Rose, 2007).

Es lo que ocurre en cláusulas como la siguiente, que aparece en el texto EE. Debido a que el MoD gira en torno al concepto de población, la frase preposicional que antecede al Tema no marcado puede ser asumida como una aclaración, y no como una orientación del contenido de la cláusula.

Para efectos legales, la población de un Estado la conforman todos sus habitantes, permanentes o transeúntes, nacionales o extranjeros (Naranjo, 2012, p. 108).

Previo a los Temas experienciales, es factible que aparezca un Tema textual o un Tema interpersonal. El primero deja explícitas las relaciones clausulares continuativas o conjuntivas, de manera que su significado no se limita al nivel gramatical, sino que trasciende hacia el estrato semántico interclausular; por ello, hacen parte de los mecanismos de cohesión que configuran al texto como unidad de sentido (Halliday y Hasan, 1989).

Para los textos objeto de estudio, $31 \%$ de las cláusulas de TN y $39 \%$ de las de TF poseen un Tema textual que pone en evidencia las relaciones lógicas construidas entre la información aportada por cada cláusula. Sin embargo, la experiencia ha demostrado que es necesario trabajar con los estudiantes no solo en visibilizar las posibilidades de aprendizaje que surgen cuando se esclarece el sentido, sino también en lograr una interpretación adecuada del significado que aportan los temas textuales. Como ocurre en el siguiente fragmento:

1. En esas fases la constitución de los antiguos fue precisándose, primero en el mundo griego y después en el romano, con caracteres cada vez más definidos, en los términos de un gran proyecto de conciliación social y política.

2. Por esto, la constitución de los antiguos nunca fue la constitución de los vencedores' (Fioravanti, 2011, p. 17).

El Tema textual "por eso" establece una relación explícita de causa- consecuencia entre las cláusulas. 
Finalmente, el Tema interpersonal ocurre en solo un $8 \%$ de las cláusulas; constituye, por lo regular, un comentario al contenido del mensaje, pero no hace parte de él; su función es orientar al interlocutor acerca del punto de vista a partir del cual entender la información en la cláusula (Thompson, 2004). En otros casos, se trata de un mecanismo de los autores para posicionarse como expertos en el tema, en tanto conocen las diferentes posturas teóricas y toman partido con las concepciones construidas por ellos mismos, retomando las posturas de otros autores. Como ocurre en la siguiente cláusula:

En verdad, la obra de Marsilio no está explícitamente dedicada al estudio del nuevo poder municipal. (Fioravanti, 2011, p. 20)

A nivel del aprendizaje, es posible afirmar que los Temas interpersonales son útiles en tanto permiten señalar información de especial relevancia en el texto:

Desde luego, es importante tomar en cuenta los criterios de diferenciación, particularmente en lo que respecta a elementos previos y elementos determinantes. (Naranjo, 2012, p. 125)

Pero interfieren en el flujo del discurso cuando se presentan como marcadores para construir la imagen de un lector experto.

\section{Congruencia}

La percepción inicial sobre la mayor complejidad de los TF se confirma cuando se comparan los datos obtenidos del estudio, a nivel de la congruencia entre la metafunción textual y su realización en los estratos semántico y léxico.

De manera congruente, el hiperTema coincide con la cláusula inicial del párrafo y permite anticipar su contenido; además, dirige la elección de los temas, que a su vez construyen el MoD. Sin embargo, en los TF, es poco frecuente encontrar cláusulas hipertemáticas y continuidad en los temas; por tanto, es el lector quien debe almacenar toda la información en la memoria de trabajo, para luego sintetizarla; asimismo, debe estar atento a la información tanto en el Tema como en el Rema, que le permita identificar el momento en el que el flujo del discurso cambia de rumbo.

Por otro lado, los patrones de realización construyen relaciones léxicas entre el Tema de una cláusula y el Rema de la cláusula precedente. Este tipo de construcciones garantiza, en cierta medida, que el elemento en posición temática corresponda con un dato conocido. Sin embargo, suele ocurrir que la información se vincule con el Rema y no con el Tema anterior, de modo que no se sostiene la orientación para irradiar desde allí otros datos relacionados con el mismo asunto; por el contrario, el contenido se desarrolla como una espiral de contenidos imbricados y relaciones lógicas, que aumentan la densidad conceptual.

A nivel de la cláusula, los TF contienen más Temas marcados que los TN. Si bien las Circunstancias de tiempo y espacio generan un campo de conocimiento compartido que facilita el aprendizaje (Montemayor, 2009), las Circunstancias de ángulo pueden ocasionar el efecto contrario. En este tipo de cláusulas, el asunto central del texto gira en torno al pensamiento del autor, no al autor en sí mismo; por tanto, es necesario empezar a reconstruir las ideas que dan cuenta de la teoría y, además, estar en la capacidad de determinar la novedad o la oposición entre distintos autores.

Por ejemplo, en la cláusula 4 del siguiente párrafo se insertan datos de autores y fechas para enfatizar en información que, en las cláusulas anteriores, habían sido presentadas en el lugar del rema, de manera que se genera una espiral de relaciones de identidad que requieren mayor capacidad en la memoria de trabajo.

1. Volvamos, por tanto, tras esta aclaración, a la doctrina política de la mitad del siglo IV. ${ }^{3}$

2. Al responder a las exigencias que antes hemos indicado, y en particular a la búsqueda de una forma de gobierno, no podía prescindir de la valoración

3 En este caso, el subrayado simple identifica la circunstancia de ángulo. 
—al mismo tiempo histórica y teórica - de la forma de gobierno que cerca de un siglo antes había caracterizado profundamente la experiencia política griega, especialmente la ateniense:

3. [La forma de gobierno] era ciertamente, como sabemos, la forma democrática.

4. En efecto, con Clístenes primero, y su reforma constitucional de 508-507, y con Pericles después, de 460 a 430, la ciudad de Atenas había adquirido una conformación desde el punto de vista político de carácter destacadamente democrático.

5. En concreto, forma democrática de gobierno significaba: primacía absoluta de la asamblea de todos los ciudadanos atenienses para la asunción de las decisiones de relevancia colectiva; derecho de palabra y de propuesta dentro de la asamblea atribuido a todo ciudadano sin discriminación alguna; extracción por suerte de los cargos públicos y de las magistraturas, comprendidos los tribunales, de nuevo sobre el presupuesto de una igualdad absoluta entre los ciudadanos, de tal manera que todos son considerados dignos de acceder incluso a los más altos cargos; alternancia anual de los gobernantes, que compromete en la responsabilidad de gobierno a la parte más tenaz posible de la ciudadanía; obligación de los mismos gobernantes de rendir cuentas públicamente.

Asimismo, los TF incluyen un gran número de referenciales como participante concordante, elementos que obligan al lector a moverse hacia delante o hacia atrás en el texto, para determinar el contenido al cual se hace mención.

Tabla 3 Niveles de congruencia, Naranjo vs. Fioravanti

\begin{tabular}{llcc}
\hline & & $\begin{array}{c}\text { Naranjo } \\
(\%)\end{array}$ & $\begin{array}{c}\text { Fioravanti } \\
(\%)\end{array}$ \\
\hline Nivel discursivo & hiperTemas* & 64 & 33 \\
& MoD & 30 & 23 \\
Nivel clausular & Circunstancia & 19 & 24 \\
& Referenciales & 27 & 31 \\
& Tema interpersonal & 7 & 15 \\
\hline
\end{tabular}

Nota: Los porcentajes de cláusulas hipertemáticas se presentan sobre el total de párrafos; los demás están basados en el total de cláusulas.
En la Tabla 3 se observan los niveles de congruencia que pueden interferir en el aprendizaje de los estudiantes.

En el estrato semántico discursivo, TN presenta mayor frecuencia de cláusulas hipertemáticas y Temas que construyen el MoD; esto permite al lector identificar el asunto del texto y las fases en el desarrollo de la información. En el estrato léxico-gramatical, TF contiene mayor número de Temas circunstanciales, que no corresponden con el asunto del texto; aparece, además, un mayor número de referencias, que demandan mayor capacidad en la memoria de trabajo. Finalmente, el Tema interpersonal construye la idea de un lector experto que no suele corresponder con el lector novel.

\section{Estrategias didácticas}

Desde la pedagogía de género (Dreyfus et al., 2016; Rose y Martin, 2012), el trabajo del docente respecto a la enseñanza del discurso especializado antes descrito consiste en poner en evidencia los distintos aspectos textuales que pueden ser tenidos en cuenta por los estudiantes para lograr el aprendizaje.

La primera tarea, previa a la lectura, es identificar el género de los textos objeto de estudio, es decir, el proceso social en el que se enmarcan: la doctrina del derecho constitucional. Al mismo tiempo, es importante que los estudiantes tengan claridad acerca de la finalidad de la lectura: acceder al contenido que sirve como base para la formación profesional en derecho. Para completar el reconocimiento del género, es necesario enfatizar en el campo del saber a abordar: el Estado y el pensamiento constitucional, así como la necesidad de ser conscientes del tenor: el texto se enmarca en el contexto de la comunidad científica, por lo que se espera del lector una serie de conocimientos previos y la capacidad para establecer relaciones lógicas entre los datos presentados. En la Tabla 4 se sintetizan estos aspectos.

Durante la lectura, la pedagogía de género recomienda iniciar con una lectura panorámica, donde se reconozcan las secciones del texto que van a permitir 
Tabla 4 Orientaciones previas a la lectura

\begin{tabular}{|c|c|c|c|c|c|}
\hline \multicolumn{2}{|c|}{ Previo a la lectura } & \multicolumn{4}{|c|}{ Capítulos } \\
\hline & & Noción de Estado & $\begin{array}{c}\text { Elementos del } \\
\text { Estado }\end{array}$ & $\begin{array}{c}\text { Constitución de los } \\
\text { antiguos }\end{array}$ & $\begin{array}{l}\text { Constitución } \\
\text { Medieval }\end{array}$ \\
\hline \multirow[t]{2}{*}{ Género } & Proceso social & \multicolumn{4}{|c|}{ Enseñanza aprendizaje del derecho constitucional } \\
\hline & $\begin{array}{l}\text { Orientado a } \\
\text { fines }\end{array}$ & $\begin{array}{l}\text { Comprender el concepto } \\
\text { de Estado }\end{array}$ & $\begin{array}{l}\text { Reconocer los } \\
\text { elementos del Estado }\end{array}$ & $\begin{array}{l}\text { Relacionar el contexto } \\
\text { sociopolítico con el } \\
\text { pensamiento constitucional } \\
\text { de la Antigijedad }\end{array}$ & $\begin{array}{l}\text { Identificar los rasgos } \\
\text { del pensamiento } \\
\text { constitucional en la Edad } \\
\text { Media }\end{array}$ \\
\hline \multirow[t]{6}{*}{ Registro } & Campo & Concepto de Estado & Elementos del Estado & $\begin{array}{l}\text { Pensamiento constitucional } \\
\text { de la Antigivedad }\end{array}$ & $\begin{array}{l}\text { Pensamiento } \\
\text { constitucional de la Edad } \\
\text { Media }\end{array}$ \\
\hline & Tenor & Escritor experto. & & \multirow{2}{*}{\multicolumn{2}{|c|}{$\begin{array}{l}\text { Escritor experto. } \\
\text { Comunidad académica que revisa las teorías relacionadas } \\
\text { con la Constitución }\end{array}$}} \\
\hline & & Lector que se inicia en el & nocimiento del derecho & & \\
\hline & Modo & \multicolumn{4}{|l|}{ Texto escrito } \\
\hline & & \multicolumn{4}{|c|}{ Lenguaje constitutivo de la actividad } \\
\hline & & \multicolumn{4}{|c|}{ Términos especializados del campo del derecho } \\
\hline
\end{tabular}

recordar el contenido como bloques de información. Por tratarse de textos macrogenéricos, estas etapas de realización no se limitan a las descritas por Rose y Martin (2012), en donde un reporte descriptivo presenta primero el elemento a describir, para pasar luego a mencionar sus características, o el reporte composicional señala la entidad y posteriormente explica sus partes. Los textos objeto de estudio, si bien pueden ser categorizados en una familia de géneros, deben ser revisados desde una perspectiva topológica, es decir, desde sus posibilidades de mezclar, cambiar y combinar estructuras dentro del mismo género (Eggins y Martin, 2003).

En TN, esta labor no presenta mayores dificultades, pues el uso de subtítulos numerados jerárquicamente permite identificar las fases en el desarrollo de la información. Por su parte, los TF requieren una estrategia diferente, ya que CA solo incluye un subtítulo al inicio del capítulo, mientras que los subtítulos en CM precisan cierto grado de inferencia para determinar la relación lógica entre ellos.

Para resolver la falta de pistas explícitas que construyen las fases del discurso, es útil incorporar la revisión del MoD, esto es, revisar la recurrencia de los ítems léxicos ubicados en posición temática, y los apartados donde se modifican. Este ejercicio permite identificar el asunto a tratar en cada sección.

Cumplido el reconocimiento de las fases que sitúan al lector en el contenido a desarrollar, es posible pasar a una lectura detallada. Esta consiste en enfocar la atención del estudiante hacia aspectos puntuales del texto que construyen el significado, señalar estos elementos, constatar que han sido comprendidos y reelaborar el contenido.

Desde la metafunción textual, la labor del maestro se enfoca en andamiar la comprensión de los ítems léxicos ubicados como Tema, principalmente, aquellos que construyen nominalizaciones, términos especializados o presentan nombres de autores. En el primer caso, es necesario desempacar la información; en el segundo y tercero, frecuentemente es indispensable aportar conocimientos del campo del derecho, necesarios para la comprensión, pero no disponibles de manera explícita en el texto. Asimismo, en la construcción del tejido discursivo, el maestro debe llamar la atención sobre los referenciales, las elisiones y los sinónimos que obligan al lector 
a recordar la información previa, de manera que el estudiante logre seguir el hilo del discurso.

Una de las tareas quizá de mayor dificultad durante la lectura detallada es enseñar a comprender las relaciones lógicas que se establecen entre las cláusulas, pues implica un ejercicio de razonamiento que supera el lenguaje como experiencia de la realidad concreta y lo acerca a la esfera del pensamiento abstracto. La comprensión de estas relaciones lógicas se hace explícita con el uso de marcadores discursivos, que deben ser subrayados y verificada su comprensión.

Finalmente, es indispensable prestar atención a los temas experienciales de tipo circunstancia y a los temas interpersonales, ninguno de los cuales, en el caso de los textos objeto de estudio, contienen el peso semántico del asunto de la cláusula. La función de las Circunstancias es crear un nexo de conocimiento compartido con el lector; los interpersonales permiten llamar la atención sobre un aspecto relevante del texto.

A manera de ilustración de las acciones a realizar durante la lectura detallada, en la Tabla 5 se presenta un segmento de CA de Fioravanti, donde se subrayan los aspectos a tener en cuenta con los estudiantes.

En el párrafo anterior, las cláusulas 1 y 2 no poseen Tema por lo que el ítem léxico que configura el asunto debe ser hallado en el lugar del Rema. Es solo en la cláusula 3 donde se evidencia explícitamente el asunto a tratar en el párrafo: el origen de la constitución desde la obra de Platón. En esta cláusula, al participante concordante le antecede una circunstancia de ángulo lo cual genera información en dos sentidos: 1) el autor, y 2) la información que será explicada. El desarrollo del contenido sobre el origen de la constitución continúa en la cláusula 4 , pero se realiza a través de una cláusula incrustada. Por su parte, la cláusula 5 presenta como participante concordante un sujeto elidido cuya referencia puede resultar ambigua ([ellos]), situación semejante ocurre en la cláusula 6 , cuyo Tema se realiza a partir de una referencia con la cual se alude al contenido de las dos cláusulas anteriores.
Las cláusulas 7 y 8 utilizan Temas concordantes entre sí, pero con términos sobre los cuales el lector debe determinar el referente. La conexión entre las cláusulas 3, 4 y 5 no se genera por conexión de Temas, sino a partir de la relación entre el Tema con un dato ubicado en el Rema de la cláusula precedente, lo cual genera una serie de contenidos imbricados.

Finalizada la lectura detallada de todo el texto, el docente titular busca que los estudiantes logren esquematizar la información. Para ello, se regresa a los datos obtenidos de la lectura panorámica, y los aprendizajes logrados en la lectura detallada; los primeros, orientarán la forma general del diagrama, mientras que los segundos proporcionarán la información clave en los niveles de menor jerarquía.

De nuevo, los TN resultan más fáciles de esquematizar, gracias al uso de subtítulos claramente jerarquizados; para el caso de TF, es necesario un acompañamiento más cercano que evidencie el MoD.

\section{Conclusiones}

El trabajo de investigación que acá se presenta ha descrito cuatro textos usados para aprender en la asignatura derecho constitucional. El análisis a partir de la LSF, específicamente desde la metafunción textual, evidencia que tanto el reconocimiento del género como los sistemas de PERIODICIDAD Y TEMA constituyen una primera fase en los procesos de alfabetización académica, en tanto que permiten reconocer el contenido global y las fases en el desarrollo de la información, lo cual aporta un marco de referencia sobre el que los estudiantes pueden partir para reconstruir los datos generales que necesitan aprender. Esto es especialmente útil si se tiene en cuenta que una de las principales actividades sugeridas por el docente disciplinar es la elaboración de esquemas que permitan sintetizar el contenido del texto.

En el nivel de la cláusula, es necesario prestar atención cuando el participante concordante carece de la carga semántica necesaria para actuar como orientador de la información y se genera entonces 
Tabla 5 Aspectos para una lectura detallada

\begin{tabular}{|c|c|c|}
\hline Cláusula & Texto & Comentario \\
\hline 1 & $\begin{array}{l}\text { Existe un último aspecto de la obra de Platón que confirma } \\
\text { todo esto. }\end{array}$ & $\begin{array}{l}\text { Nexo con el contenido del párrafo anterior. Anticipa la } \\
\text { orientación del párrafo: la obra de Platón. } \\
\text { Requiere haber comprendido la información previa } \\
\text { señalada por el demostrativo "esto". }\end{array}$ \\
\hline 2 & $\begin{array}{l}\text { Se trata del aspecto siempre decisivo que versa sobre la } \\
\text { problemática del origen de la constitución. }\end{array}$ & $\begin{array}{l}\text { Especifica la orientación del contenido: el origen de la } \\
\text { constitución. }\end{array}$ \\
\hline 3 & $\begin{array}{l}\text { Para Platón, la constitución que ha tenido un origen violento } \\
\text { está destinada a decaer en sequida. }\end{array}$ & $\begin{array}{l}\text { Cláusula hipertemática, aunque no se encuentra al } \\
\text { principio del párrafo, permite sintetizar todo el contenido. }\end{array}$ \\
\hline 4 & $\begin{array}{l}\text { Es lo que sucedió, de nuevo, con la incierta constitución } \\
\text { democrática, que desde el principio sólo fue la constitución } \\
\text { de los vencedores, principalmente de los pobres, que } \\
\text { inmediatamente después de conquistar el poder mataron a una } \\
\text { parte de sus adversarios, }\end{array}$ & $\begin{array}{l}\text { Desarrollo del contenido de la cláusula anterior. } \\
\text { El participante concordante es toda la cláusula incrustada. }\end{array}$ \\
\hline 5 & $\begin{array}{l}\text { es decir, de la minoría más rica y acomodada, desterraron a otros e } \\
\text { hicieron upartícipes a los demás del gobierno y de las magistraturas". }\end{array}$ & $\begin{array}{l}\text { El conector "es decir" pone en evidencia que esta cláusula } \\
\text { explicará la información de la anterior. }\end{array}$ \\
\hline 6 & Pero esto no sólo sirve para la democracia: & $\begin{array}{l}\text { El conector no expresa una relación simple de oposición; al } \\
\text { continuar con la lectura de la cláusula, se puede identificar } \\
\text { una relación de adición: el ejemplo sirve para otros casos. }\end{array}$ \\
\hline 7 & $\begin{array}{l}\text { [esto] es válido para todos los casos en los que "los vencedores } \\
\text { se adueñan de tal manera de la vida del Estado que no dejen ni } \\
\text { más pequeña parte de responsabilidad a los vencidos, ni a ellos } \\
\text { ni a sus descendientes". }\end{array}$ & Incluye otro caso que permite respaldar la idea central. \\
\hline 8 & En estos casos, lo que resulta "no son constituciones". & $\begin{array}{l}\text { Reafirma la consecuencia de los casos donde el poder } \\
\text { sobre el Estado tiene un origen violento: no se puede } \\
\text { hablar de una constitución. } \\
\text { Utiliza un tema circunstancial para generar la conexión } \\
\text { con las ideas anteriores y de nuevo, la cláusula incrustada } \\
\text { como participante concordante. }\end{array}$ \\
\hline
\end{tabular}

un $\mathrm{MoD}$ que no se construye hacia un mismo aspecto, sino que se teje con ideas imbricadas (a la manera de una matrioska) que producen mayor densidad en el contenido del texto. Del mismo modo, revisar los casos donde el Tema marcado genera una interrupción en el flujo del discurso en tanto antecede al participante. Para el caso de las cláusulas donde el participante realiza el $\mathrm{MoD}$, es necesario observar los numerosos casos donde este se construye a partir de nominalizaciones o referencias; las primeras, aumentan el nivel de abstracción y la necesidad de conocimientos previos, las segundas requieren moverse en el texto para determinar el significado del referente. Asimismo, es necesario revisar el papel de los Temas textuales e interpersonales que aportan a la comprensión cuando evidencian las relaciones lógicas entre las ideas o los aspectos relevantes del texto, pero que se deben trabajar de manera explícita en el aula.

De otro lado, queda pendiente, ampliar la reflexión en torno al papel del elemento en posición temática para el caso del español. Al tratarse de una lengua con mayor libertad sintáctica, se distancia de los resultados en inglés, donde es más frecuente que exista una relación de realización entre el hiperTema o el Tema 
y el asunto del párrafo o cláusula, respectivamente; por el contrario, como lo demuestran los resultados de esta investigación, no parece existir el mismo nivel de recurrencia en español.

\section{Agradecimientos}

Un sincero reconocimiento a la maestra Estela Moyano por su acompañamiento a lo largo de este proceso investigativo.

\section{Referencias}

Álvarez, S., Benítez, T. y Rosado, N. (2018). Español académico para aprender en las disciplinas. Revista Española de Lingüistica Aplicada, 12(24), 32-55. https:// doi.org/10.26378/rnlael122438

Carlino, P. (2013). Alfabetización académica diez años después. Revista Mexicana de Investigación Educativa, 18(57), 355-381.

Dreyfus, S., Humphrey, S., Mahboob, A. y Martin, J. R. (2016). Genre pedagogy in higher education. The SLATE Project. Palgrave Macmillan. https://doi. org/10.1007/978-1-137-31000-2

Eggins, S. (2002). Introducción a la lingüistica sistémica. Universidad de la Rioja.

Eggins, S. y Martin, J. (2003). El contexto como género: una perspectiva lingüística funcional. Revista Signos, 36(54), 185-205. https://doi.org/10.4067/ S0718-09342003005400005

Fioravanti, M. (2011). Constitución. De la Antigüedad a nuestros dias. Trotta.

Fries, P. (1983). On the status of theme in English: Arguments from discourse. En J. Petöfi y E. Sözer (Eds.), Micro and macro connexity of text (pp. 116-152). Helmut BuskeVerlag.

García-Parejo, I., Ahern, A. y García-Bermejo, L. (2017). La "pedagogía de los géneros discursivos" en grados de formación del profesorado: presentación de dos proyectos de innovación docente. Lenguaje y textos, (46), 69-80. https://doi.org/10.4995/lyt.2017.8740

Halliday, M. (1993). Towards a language-based theory of learning. Linguistics and Education, 5(2), 93-116. https://doi.org/10.1016/0898-5898(93)90026-7

Halliday, M. (2014). Halliday's introduction to functional grammar. Routledge.

Cómo citar este artículo: Rojas, I. (2021). Metafunción textual: base para la alfabetización académica en derecho constitucional. Íkala, Revista de Lenguaje y Cultura, 26(1), 167-182. https://doi.org/10.17533/ udea.ikala.v26n01a01
Halliday, M. y Hasan, R. (1989). Language, context and text: aspects of language in a social-semioticperspective. $\mathrm{Ox}$ ford University Press.

Halliday, M. y Martin, J. (1993). Writing science. Literacy and discursive power. Burgess Science Press.

Ignatieva, N. y Rodríguez, D. (Coords). (2016). Lingüistica sistémico-funcional en México: aplicaciones e implicaciones. UNAM.

Martin, J. (1992). English text. System and structure. John Benjamins. https://doi.org/10.1075/z.59

Martin, J. (2016). One of three traditions: Genre, functional linguistics and the Sydney School. En N. Artemeva y A. Freedman (Eds.), Genre studies around the globe. Beyond the three traditions (pp. 31-77). Trafford.

Martin, J. y Rose, D. (2007). Working with discourse. Meaning beyond the clause. Continuum.

Martin, J. y Rose, D. (2008). Genre relations, mapping culture. Equinox.

Montemayor, A. (2009). Tema: una perspectiva funcional de la organización del discurso. Eudeba.

Moyano, E. I. (2007). Enseñanza de habilidades discursivas en español en contexto pre-universitario: Una aproximación desde la LSF. Signos, 40(65), 573-608. https://doi.org/10.4067/ S0718-09342007000300009

Moyano, E. I. (2010) Escritura académica a lo largo de la carrera: un programa institucional. Revista Signos, 43(74), 465-488.

Moyano, E. I. (2016). Theme in English and Spanish: Different means of realization for the same textual function. English Text Construction, 9(1), 190-219. https://doi. org/10.1075/etc.9.1.10moy

Naranjo, V. (2012). Teoría constitucional e instituciones politicas. Temis.

Narvaja de Arnoux, E., Di Stefano, M. y Pereira, C. (2004). La lectura y la escritura en la universidad. Universidad de Buenos Aires.

Rose, D. y Martin, J. (2012). Learning to write, reading to learn. Equinox. [Traducción al español, 2018, Leer para aprender, lectura y escritura en las áreas del curriculo. Pirámide].

Thompson, G. (2004). Introducing functional grammar. Oxford University Press. 